\title{
Kinesio Taping no Tratamento de Mulheres com Linfedema Pós-Mastectomia: Revisão Narrativa
}

\author{
Rejeane Santos da Conceição, ${ }^{1}$ Daniela Débora Santos de Oliveira ${ }^{2}$
}

\section{RESUMO}

O linfedema é uma doença crônica que gera acúmulo de linfa e pode acometer mulheres que se submeteram ao tratamento do câncer de mama, ocorrendo diminuição do fluxo no sistema linfático e acúmulo da linfa no espaço intersticial. O Kinesio Taping (KT) demonstra ser uma técnica eficaz no tratamento para a redução do volume do linfedema em mulheres mastectomizadas. Este estudo trata-se de uma revisão da literatura do tipo narrativa, realizada por meio de pesquisa em base de dados e em bibliotecas virtuais: Pubmed, Lilacs, Medline, Scielo e Bireme nos idiomas inglês e português. As evidências disponíveis permitem concluir que o KT, associado a outras técnicas, demostra uma redução do linfedema, trazendo benefícios às mulheres com linfedema pós-mastectomia.

Palavras-chave: Kinesio taping. Mastectomia. Linfedema.

\section{KINESIO TAPING IN THE TREATMENT OF WOMEN WITH LYMPHEDEMA AFTER MASTECTOMY: NARRATIVE REVIEW}

\section{ABSTRACT}

Lymphedema is a chronic disease that generates lymph tumors and can affect women who have undergone breast cancer treatment, with decreased flow in the lymphatic system and increased lymph in the interstitial space. Kinesio Taping (KT) proves to be an effective treatment technique to reduce the volume of lymphedema in mastectomized women. This study deals with a review of the narrative literature carried out through database and virtual libraries research: Pubmed, Lilacs, Medline, Scielo and Bireme in English and Portuguese. As the available ones allow us to conclude what KT associated with other techniques demonstrated as a reduction of lymphedema, it brings benefits as women with lymphedema after mastectomy.

Keywords: Kinesio taping. Mastectomy. Lymphedema.

RECEBIDO EM: 3/7/2019

MODIFICAÇÕES SOLICITADAS EM: 24/6/2020

ACEITO EM: 29/12/2020

\footnotetext{
${ }^{1}$ Autora correspondente. Faculdade Regional da Bahia - Unirb. Av. Tamburugy, 474 - Patamares. Salvador/BA, Brasil. CEP 41680 440. http://lattes.cnpq.br/3088053633039861. https://orcid.org/0000-0001-5013-2157. rejeane.conceicao@unirb.edu.br

2 Faculdade Regional da Bahia - Unirb. Salvador/BA, Brasil.
} 


\section{INTRODUÇÃO}

Segundo o Instituto Nacional de Câncer (Inca), atualmente o câncer de mama é o segundo mais diagnosticado no mundo, tornando-se um problema de saúde pública em razão do aumento de novos casos. No Brasil, o atraso no diagnóstico e no início do tratamento varia nas diferentes regiões do país, e essas variações têm relação direta com a qualidade do prognóstico (INCA, 2018).

A mastectomia é o procedimento cirúrgico utilizado como a principal forma de tratamento do câncer de mama, podendo ser realizada de forma parcial ou total. A parcial (quadrantectomia) é caracterizada como procedimento de retirada de um quadrante da mama, tendo como indicação tumores de até três centímetros, dependendo da sua localização; a total ou radical é quando se dá a retirada de todo o tecido mamário que está sendo acometido. Em situações onde ocorre comprovação de células tumorais além dos limites da mama (músculo e linfonodos), é necessário um procedimento cirúrgico mais extenso e com área de dissecção maior, que pode resultar em complicações mesmo após o final do tratamento para o câncer (BRASIL, 2018; PACHECO; COSTA; HADDAD, 2018).

Quando se realiza a dissecção axilar são retirados linfonodos axilares dessa região após confirmação realizada por meio da biopsia do Linfonodo Sentinela. Esse procedimento é necessário e importante para a continuidade do tratamento para evitar o retorno do tumor, mas causa alterações do fluxo linfático que resulta em aumento da pressão hidrostática na parede dos vasos, ocorrendo dilatação e afastamento das válvulas linfáticas, o que provoca o linfedema (OLIVEIRA et al., 2015).

Segundo a Sociedade Brasileira de Angiologia e de Cirurgia Vascular (SBACV), o linfedema é uma doença crônica que gera acúmulo de linfa nos espaços intersticiais em virtude de alteração do sistema linfático. É considerada a complicação mais comum após a mastectomia, e pode causar outras consequências, como a redução de força muscular, a diminuição da amplitude de movimento, dor e o aumento do peso no membro superior afetado, transformando-se em uma condição crônica (SILVA et al., 2016; TACANI et al., 2012).

A fisioterapia atua no tratamento do linfedema pós-mastectomia, com o objetivo principal de estimular a circulação linfática, promover uma cicatrização mais rápida e preservar/restaurar o sistema cinético-funcional do membro superior afetado. A Terapia Complexa Descongestiva (TCD) consiste na combinação de várias técnicas que atuam conjuntamente, dependendo da fase em que se encontra o linfedema, e inclui a drenagem linfática manual (DLM), cuidados gerais com a pele, cinesioterapia, enfaixamento compressivo por luvas/braçadeiras, sendo a técnica mais recente o kinesio taping (PINHEIRO; GODOY; SUNEMI, 2015; TACANI et al., 2012).

O Kinesio taping (KT) é uma bandagem elástica de algodão com adesivo acrílico que pode ser esticado até $140 \%$ do seu comprimento original, criada pelo quiropráta japonês Kenzo Kase, com o objetivo de criar uma fita terapêutica que pudesse suportar articulações e músculos, sem restringir a amplitude de movimento (CSAPO, ALEGRE, 2014).

Editora Unijuí - Revista Contexto \& Saúde - ISSN 2176-7114 - v. 21, n. 42, jan./jun. 2021 
Com é uma técnica relativamente nova no tratamento do linfedema, o KT pode ser aplicado em conjunto com outras técnicas pós-mastectomização, pois melhora a circulação da linfa nos vasos em virtude da redução da pressão no sistema linfático. Nessa técnica linfática, as fitas são aplicadas em forma de espiral ou cruzadas da região proximal para as distais, melhorando a amplitude de movimento, proporcionando estímulo sensorial e com a vantagem de ser uma abordagem não invasiva e indolor (TARADAJ et al., 2014).

O objetivo deste estudo foi revisar, mediante a literatura científica, a efetividade do KT no tratamento do linfedema pós-mastectomia.

\section{MÉTODOS}

O presente estudo é uma revisão narrativa de literatura, objetivando expor os efeitos do KT no tratamento do linfedema pós-mastectomização. Foram utilizados artigos de base de dados buscados nas bibliotecas virtuais Pubmed, Lilacs, Medline, Scielo e Bireme, nos idiomas inglês e português, e empregadas as seguintes palavras-chave: kinesio taping, Mastectomia e Linfedema. O período de publicação não foi um critério utilizado em razão da pouca publicação em relação ao tema. Os critérios de inclusão foram títulos disponíveis na íntegra, relacionados com o tema proposto, nos idiomas português e inglês, e os critérios de exclusão foram títulos repetidos e os que não se aplicavam ao tema.

Segundo Ferenho e Fernandes et al. (2016), as revisões narrativas são publicações amplas, apropriadas para descrever e discutir o desenvolvimento de um determinado assunto, sob o ponto de vista teórico ou contextual. São textos que instituem a análise da literatura científica na explicação e análise crítica do autor.

\section{RESULTADOS E DISCUSSÃO}

Após a coleta de dados, foram inclusos sete artigos: - um em português e seis em inglês.

Os artigos revisados estão organizados, na Tabela 1, pelo ano de publicação, autores, títulos, objetivos, tipo de estudo e principais resultados encontrados. Foram considerados relevantes para o estudo o efeito do KT, o método de aplicação, a utilização para avaliação do linfedema e os resultados encontrados.

Tabela 1 - Artigos revisados organizados pelo ano de publicação, autores, títulos, objetivos, tipo de estudo e principais resultados

\begin{tabular}{|l|l|l|l|}
\hline $\begin{array}{l}\text { Título/autor(es)/ } \\
\text { ano }\end{array}$ & \multicolumn{1}{|c|}{ Objetivos } & \multicolumn{1}{c|}{$\begin{array}{c}\text { Tipo de } \\
\text { estudo }\end{array}$} & \multicolumn{1}{c|}{ Principais achados } \\
\hline $\begin{array}{l}\text { Kinesio Taping } \\
\text { associado à } \\
\text { drenagem } \\
\text { linfática manual } \\
\text { no linfedema } \\
\text { pós-mastectomia } \\
\text { (PINHEIRO; } \\
\text { GODOY; SUNEMI, } \\
\text { 2015). }\end{array}$ & $\begin{array}{l}\text { Verificar o efeito da } \\
\text { aplicação do Kinesio } \\
\text { taping associado à } \\
\text { drenagem linfática } \\
\text { manual no tratamento } \\
\text { do linfedema. }\end{array}$ & $\begin{array}{l}\text { Estudo de } \\
\text { caso }\end{array}$ & $\begin{array}{l}\text { A aplicação do Kinesio ta- } \\
\text { ping reduziu o linfedema, } \\
\text { aumentou a motivação, } \\
\text { teve melhor adesão que } \\
\text { do enfaixamento com- } \\
\text { dressivo, menor dificulda- } \\
\text { de no uso e maior confor- } \\
\text { to e maior facilidade na } \\
\text { execução das atividades } \\
\text { de vida diária. }\end{array}$ \\
\hline
\end{tabular}

Editora Unijuí - Revista Contexto \& Saúde - ISSN 2176-7114 - v. 21, n. 42, jan./jun. 2021 


\begin{tabular}{|c|c|c|c|}
\hline $\begin{array}{l}\text { Safety and } \\
\text { tolerability of } \\
\text { Kinesio }{ }^{\circledR} \text { Taping } \\
\text { in patients with } \\
\text { arm lymphedema: } \\
\text { medical device } \\
\text { clinical study } \\
\text { (MARTINS et al., } \\
\text { 2015). }\end{array}$ & $\begin{array}{l}\text { Avaliar a segurança } \\
\text { e tolerabilidade do } \\
\text { Kinesio }{ }^{\circledR} \text { Taping (KT) } \\
\text { em pacientes com } \\
\text { linfedema. }\end{array}$ & $\begin{array}{l}\text { Estudo } \\
\text { clínico não } \\
\text { randomizado }\end{array}$ & $\begin{array}{l}\text { O KT mostrou-se seguro } \\
\text { e tolerável, com melhora } \\
\text { da funcionalidade } \\
\text { e nenhum paciente } \\
\text { apresentou lesões } \\
\text { cutâneas. }\end{array}$ \\
\hline $\begin{array}{l}\text { The influence of } \\
\text { Kinesiology Taping } \\
\text { on the volume of } \\
\text { lymphoedema and } \\
\text { manual dexterity } \\
\text { of the upper } \\
\text { limb in women } \\
\text { after breast } \\
\text { cancer treatment } \\
\text { (TARADAJ et al., } \\
\text { 2015). }\end{array}$ & \begin{tabular}{|l|} 
Avaliar o efeito da \\
Kinesio Taping (KT) no \\
tamanho do linfedema \\
e agilidade manual \\
do membro superior \\
em mulheres após \\
tratamento de câncer \\
de mama.
\end{tabular} & $\begin{array}{l}\text { Estudo } \\
\text { Randomizado }\end{array}$ & $\begin{array}{l}\text { O KT não é um método } \\
\text { eficaz para a redução } \\
\text { do linfedema II e III, } \\
\text { não podendo substituir } \\
\text { o enfaixamento de } \\
\text { multicamadas. }\end{array}$ \\
\hline $\begin{array}{l}\text { The influence of } \\
\text { Kinesiology Taping } \\
\text { on the reduction } \\
\text { of lymphoedema } \\
\text { among women } \\
\text { after mastectomy } \\
\text {-preliminary study } \\
\text { (POP et al., 2014). }\end{array}$ & $\begin{array}{l}\text { Avaliar a influência do } \\
\text { Kinesio Taping sobre a } \\
\text { redução do linfedema } \\
\text { entre mulheres após a } \\
\text { mastectomia. }\end{array}$ & $\begin{array}{l}\text { Estudo } \\
\text { Randomizado }\end{array}$ & $\begin{array}{l}\text { A técnica de Kinesio } \\
\text { Taping exerceu influência } \\
\text { na melhoria da } \\
\text { mobilidade e articulação } \\
\text { dos membros superiores } \\
\text { e a força de preensão. }\end{array}$ \\
\hline $\begin{array}{l}\text { Kinesiology } \\
\text { Taping reduces } \\
\text { lymphedema } \\
\text { of the upper } \\
\text { extremity in } \\
\text { women after } \\
\text { breast cancer } \\
\text { treatment: a pilot } \\
\text { study (MALICKA et } \\
\text { al., 2014). }\end{array}$ & \begin{tabular}{|l|} 
Avaliar os efeitos das \\
aplicações de KT na \\
extensão do linfedema \\
da extremidade \\
superior em mulheres \\
após o tratamento do \\
câncer.
\end{tabular} & Estudo piloto & $\begin{array}{l}\text { Redução da extensão do } \\
\text { linfedema significativa } \\
\text { com o uso do KT, sendo } \\
\text { necessários ensaios } \\
\text { clínicos com um } \\
\text { tamanho apropriado que } \\
\text { compara os diferentes } \\
\text { modos de bandagem } \\
\text { no mesmo tipo de } \\
\text { pacientes com o mesmo } \\
\text { grau de linfedema, para } \\
\text { estabelecer o melhor } \\
\text { método de contenção, } \\
\text { dependendo das } \\
\text { características do edema. }\end{array}$ \\
\hline $\begin{array}{l}\text { Evaluation of the } \\
\text { effectiveness of } \\
\text { kinesio taping } \\
\text { application in a } \\
\text { patient with sec- } \\
\text { ondary lymphede- } \\
\text { ma in breast can- } \\
\text { cer: a case report } \\
\text { (TARADAJ et al., } \\
\text { 2014). }\end{array}$ & $\begin{array}{l}\text { Avaliar o efeito da } \\
\text { aplicação do KT no } \\
\text { linfedema secundário } \\
\text { ao câncer de mama. }\end{array}$ & $\begin{array}{l}\text { Estudo de } \\
\text { caso }\end{array}$ & $\begin{array}{l}\text { Não apresentou alergia } \\
\text { cutânea após o uso de } \\
\text { fitas Kinesio taping, a } \\
\text { terapia pareceu segura } \\
\text { e bem-sucedida e a } \\
\text { redução de volume do } \\
\text { membro foi mais rápida } \\
\text { depois do Kinesio taping. }\end{array}$ \\
\hline
\end{tabular}




\begin{tabular}{|c|c|c|c|}
\hline $\begin{array}{l}\text { Effect of } \\
\text { Kinesiology } \\
\text { Taping on Breast } \\
\text { Cancer-Related } \\
\text { Lymphedema: } \\
\text { A Randomized } \\
\text { Single-Blind } \\
\text { Controlled Pilot } \\
\text { Stud (SMYKLA et } \\
\text { al., 2013). }\end{array}$ & $\begin{array}{l}\text { Avaliar a eficácia do } \\
\text { Kinesio Taping (KT) } \\
\text { para o tratamento do } \\
\text { linfedema relacionado } \\
\text { ao câncer de mama. }\end{array}$ & $\begin{array}{l}\text { Estudo } \\
\text { randomizado }\end{array}$ & $\begin{array}{l}\text { Resultados em } \\
\text { pacientes submetidos } \\
\text { a Kinesio taping foram } \\
\text { semelhantes aos obtidos } \\
\text { no single-blind grupo } \\
\text { placebo, mas houve } \\
\text { redução do membro. }\end{array}$ \\
\hline
\end{tabular}

Fonte: Elaborada pelas autoras.

Entre os artigos selecionados, os autores destacaram o uso do kinesio Taping no tratamento de mulheres com linfedema pós-mastectomia para a melhora da qualidade de vida, restabelecimento da capacidade funcional e maior adesão ao tratamento.

Segundo Táboas et al. (2013), o linfedema começa a progredir a partir do momento em que o sistema linfático deixa de transportar substâncias que são drenadas por ele. É classificado de acordo com os graus, facilitando o diagnóstico. Grau I: linfedema que reduz com o repouso durante 24 horas e 48 horas; Grau II: linfedema que não regride após repouso do membro durante período prolongado; e Grau III: linfedema irreversível e mais grave, aspecto elefantiásico, deposição de fibrose acentuada no tecido subcutâneo e a pele adquire uma consistência dura.

Malicka et al. (2014) realizaram um estudo piloto com mulheres em pós-operatório tardio de mastectomia e foram diagnosticadas com linfedema secundário de grau I, divididas em dois grupos. Quando se realiza a comparação entre os Grupos A (KT) e B (controle) observa-se que no grupo KT houve uma redução significativa na extensão do linfedema, se mostrando um método eficaz no estágio inicial do edema e um método seguro para as mulheres, o que não ocorreu com o grupo controle.

Taradaj et al. (2015), em seu estudo randomizado, analisaram grupos de mulheres que apresentaram linfedema de grau II e III. O Grupo A foi submetido ao uso do KT e pneumáticos, Grupo B a sessões de drenagem linfática e pneumático e Grupo $C$ a intervenções fisioterapêuticas tradicionais que são drenagem linfática manual, pneumático e bandagem multicamadas. No período de quatro semanas de tratamento foi evidenciado pelos autores que o KT não é um método eficaz ou alternativo na redução do linfedema, sendo demostrada a necessidade do uso de bandagem multicamadas e a permanência conjunta da intervenção fisioterapêutica tradicional no tratamento do linfedema. Os resultados do presente estudo confirmam os dados já obtidos por outros autores, como Smykla et al. (2013). Os autores concluíram que o KT parece ser ineficaz no tratamento do linfedema pós-mastectomia nos referidos graus e não deve ser uma terapia de escolha no tratamento dos mesmos.

Pop et al. (2014) dividiram sua amostra em dois grupos em um estudo randomizado, cujo objetivo foi comparar os métodos de aplicação do KT, na qual a te-

Editora Unijuí - Revista Contexto \& Saúde - ISSN 2176-7114 - v. 21, n. 42, jan./jun. 2021 
rapia realizou-se no período de 21 dias, ocorrendo aplicação três vezes a cada 7 dias, sendo feitas medições antes e depois de cada aplicação no membro acometido. Depois da comparação entre os grupos no período estabelecido, foi confirmado que com o uso do KT ocorreu redução do linfedema e que este exerceu melhoria na mobilidade das articulações e de força de preensão, favorecendo o retorno venoso.

Pinheiro, Godoy e Sunemi (2015) realizaram um estudo do tipo relato de caso com uma mulher de 56 anos submetida ao tratamento cirúrgico de mastectomia total com esvaziamento axilar, que realizou dez sessões de fisioterapia que consistiram em DLM e aplicação do KT. Já Taradaj et al. (2015) realizaram um estudo do mesmo tipo com uma mulher de 62 anos com linfedema pós-mastectomia, submetida, no seu tratamento, a 12 sessões de DLM, compressões pneumáticas e 3 aplicações com KT. Os estudos concordam que ao final das sessões foi observado que o KT resultou em um efeito significativo na diminuição na circunferência do membro acometido e melhora na sensação de peso no membro, além do conforto e facilidade nas atividades de vida diária (AVD).

No estudo clínico não randomizado, realizado por Martins et al. (2015) em mulheres com linfedema secundário a mastectomia, no início do tratamento realizou-se a retirada da roupa de compressão e foi aplicada a técnica de KT durante o período de quatro semanas. As mulheres apresentaram baixa incidência de alteração dérmica e relataram boa tolerância ao uso do KT, mostrando-se uma técnica segura e tolerável em pacientes com linfedema, com melhora da funcionalidade e sem alteração do volume do membro afetado. O relato dos autores neste estudo confirma que KT tem a melhor aceitação e os benefícios incluíram tempo de uso, menor dificuldade na utilização, conforto e conveniência entre as mulheres com linfedema pós-mastectomia.

Até o presente momento nenhum estudo avaliou os efeitos do KT em longo prazo. Se acrescido, poderá mostrar seus benefícios às mulheres com linfedema, devendo, ainda, ser avaliados os benefícios com outras técnicas fisioterapêuticas.

\section{CONSIDERAÇÕES FINAIS}

Para os profissionais da fisioterapia na área da reabilitação vascular, recentemente foi introduzida a utilização do KT, como mostram os resultados dos estudos descritos nesta revisão narrativa de literatura, no entanto se fazem necessárias mais pesquisas para estabelecer essa conduta como opção de tratamento, pois existem poucos artigos científicos relacionados ao tema, principalmente estudos que avaliem a efetividade do KT em longo prazo como técnica de tratamento único em comparação à associação com outras técnicas de terapia descongestiva.

É possível concluir que, de acordo com os graus de classificação para o linfedema, foi evidenciado que no Grau I o KT demonstrou redução significante do membro afetado, porém no Grau II e III o enfaixamento compressivo mostrou-se ser a conduta mais eficaz na redução do linfedema, tanto de forma isolada quanto associada a outras técnicas, ocorrendo melhor adesão à terapia, menor dificuldade no uso e maior conforto e facilidade na execução das atividades de vida diária, tornando-se o Kinesio Taping uma alternativa para o tratamento.

Editora Unijuí - Revista Contexto \& Saúde - ISSN 2176-7114 - v. 21, n. 42, jan./jun. 2021 


\section{REFERÊNCIAS}

BRASIL. Ministério da Saúde. Câncer de mama: sintomas, tratamentos, causas e prevenção. 2018. Disponível em: https://saude.gov.br/saude-de-a-z/cancer-de-mama. Acesso em: 13 maio 2019.

CSAPO, R.; ALEGRE, L. M. Effects of Kinesio ${ }^{\circledR}$ taping on skeletal muscle strength. A meta-analysis of current evidence. Journal of Science and Medicine in Sport, v. 18, n. 4, 2014. FERENHO, F. H. A.; FERNANDES, R. F. Desmistificando a revisão de literatura como base para redação científica: método ssf. Revista ACB: Biblioteconomia em Santa Catarina, Florianópolis, v. 21, n. 3, p. 550-563, ago./nov. 2016.

INCA. Instituto Nacional de Câncer José Alencar Gomes da Silva. Ministério da Saúde. Rio de Janeiro, 2018. Disponível em: https://www.inca.gov.br/numeros-de-cancer. Acesso em: 5 maio 2019.

MALICKA, I. et al. Kinesiology Taping reduces lymphedema of the upper extremity in women after breast cancer treatment: a pilot study. Prz Menopauzalny, v. 13, n. 4, 2014. MARTINS, J. C. et al. Safety and tolerability of Kinesio ${ }^{\circledR}$ Taping in patients with arm lymphedema: medical device clinical study. Support Care Cancer, v. 24, n. 3, 2015.

OLIVEIRA, M. M. F. et al. Compensações linfáticas no pós-operatório de câncer de mama com dissecção axilar. J. Vasc. Bras., v. 14, n. 2, 2015.

PACHECO, F. Y. R.; COSTA, M. J. S.; HADDAD, C. A. S. Terapia física complexa no tratamento do linfedema maligno. Rev. Soc. Bras. Clin. Med., São Paulo, v. 16, n. 4, p. 238-240, out./dez. 2018. Disponível em: http://www.sbcm.org.br/ojs3/index.php/rsbcm/article/ view/379/341. Acesso em: abr. 2019.

PINHEIRO, M. D. S.; GODOY, A. C.; SUNEMI, M. M. O. Kinesio Taping associado à drenagem linfática manual no linfedema pós-mastectomia. Rev. Fisioter. S. Fun., Fortaleza, v. 4, n. 1, 2015.

POP, T. B. et al. The influence of Kinesiology Taping on the reduction of lymphoedema among women after mastectomy - preliminary study. Contemp Oncol (Pozn), 18 (2), p. 124-129, 2014.

SILVA, M. et al. Linfedema primário em membro superior esquerdo em paciente idosa. Revista Ciências em Saúde, v. 6, n. 3, 2016.

SMYKLA, A. et al. Effect of Kinesiology Taping on breast cancer-related lymphedema: a randomized single-blind controlled pilot study. Bio Med Research International, v. 2013. Disponível em: hindawi.com/journals/bmri/2013/767106/. Acesso em: 20 maio 2019.

TÁBOAS, M. I. et al. Linfedema: revisão e integração de um caso clínico. Revista da Sociedade Portuguesa de Medicina Física e de Reabilitação, Portugal, v. 23, n. 1, 2013.

TACANI, P. M. et al. Abordagem fisioterapêutica do linfedema bilateral de membros inferiores. Fisioter Mov., v. 25, n. 3, p. 561-570, 2012.

TARADAJ, J. et al. Evaluation of the effectiveness of kinesio taping application in a patient with secondary lymphedema in breast cancer: a case report. Przeglad Menopauzalny/ Menopause Review, 18(1), 2014.

TARADAJ. J. et al. The influence of Kinesiology Taping on the volume of lymphoedema and manual dexterity of the upper limb in women after breast cancer. European Journal of Cancer Care, v. 25, n. 4, p. 647-60, 2015. 